\title{
X-ray emission properties of BLAGN in the XMM-2dF Wide Angle Survey
}

\author{
S. Mateos, ${ }^{1}$ M. G. Watson, ${ }^{1}$ J. A. Tedds,${ }^{1}$ F. J. Carrera,${ }^{2}$ \\ M. Page ${ }^{3}$ and A. Corral ${ }^{2}$ \\ ${ }^{1}$ Department of Physics and Astronomy, University of Leicester, LE1 7RH, UK \\ ${ }^{2}$ Instituto de Física de Cantabria (CSIC-UC), Avenida de los Castros, 39005 Santander, Spain \\ ${ }^{3}$ Mullard Space Science Laboratory, UCL, Holmbury St. Mary, Surrey, RH5 6NT, UK \\ email: sm279@star.le.ac.uk
}

\begin{abstract}
We present the preliminary results of the X-ray spectral analysis of one of the largest samples of X-ray selected BLAGN assembled so far from the XMM-2dF Wide Angle Survey. The sample, with 641 spectroscopically identified BLAGN, provides a unique resource to carry out a statistical analysis of the emission properties of these objects over a broad range of X-ray luminosities and redshifts. The X-ray spectra of the majority of the objects were best fitted with a power law with a near constant mean spectral photon index. No obvious trend of this spectral parameter with X-ray luminosity or redshift was found.

We measured the mean photon index of our objects to be $\sim 1.96 \pm 0.05$ with an intrinsic dispersion $\sigma=0.22 \pm 0.03$. X-ray absorption was detected in $\sim 8 \%$ of the sources, with no preferred luminosity or redshift and having typical values of the absorbing column density $\leqslant 10^{22} \mathrm{~cm}^{-2}$.
\end{abstract}

Keywords. X-rays: general - X-ray surveys - galaxies: active

\section{Introduction}

The Chandra and XMM-Newton deepest pencil beam surveys have resolved more than $80 \%$ of the Cosmic X-ray background (XRB) into discrete objects confirming the discrete nature of this cosmic emission (see for example Tozzi et al. 2006) and that the XRB is dominated by Active Galactic Nuclei (AGN). Extensive statistical X-ray spectral studies of the sources detected in these surveys are providing valuable information on the nature and cosmic evolution of the population of faint AGN, however many of these results are limited by the lack of large enough samples of objects. In order to understand the accretion history in the Universe, a good knowledge of the X-ray emission properties of the population of AGN and their dependence with the luminosity and redshift of the objects is necessary.

One of the open questions that still needs to be addressed is the existing correlation between X-ray absorption and optical obscuration in AGN. AGN unification models have been successful in explaining the observational differences between different classes of AGN, however X-ray spectral analyses show that 10\% of BLAGN are absorbed in Xrays (see Mateos et al. 2005b), questioning the validity of AGN unification models in these objects. Shallower but wide area surveys are an important complement to pencil beam surveys, as they are more efficient in compiling large samples of sources at intermediate fluxes where the bulk of the XRB emission originates. Hence these surveys are an ideal database to study some of the remaining open questions regarding the AGN population. 

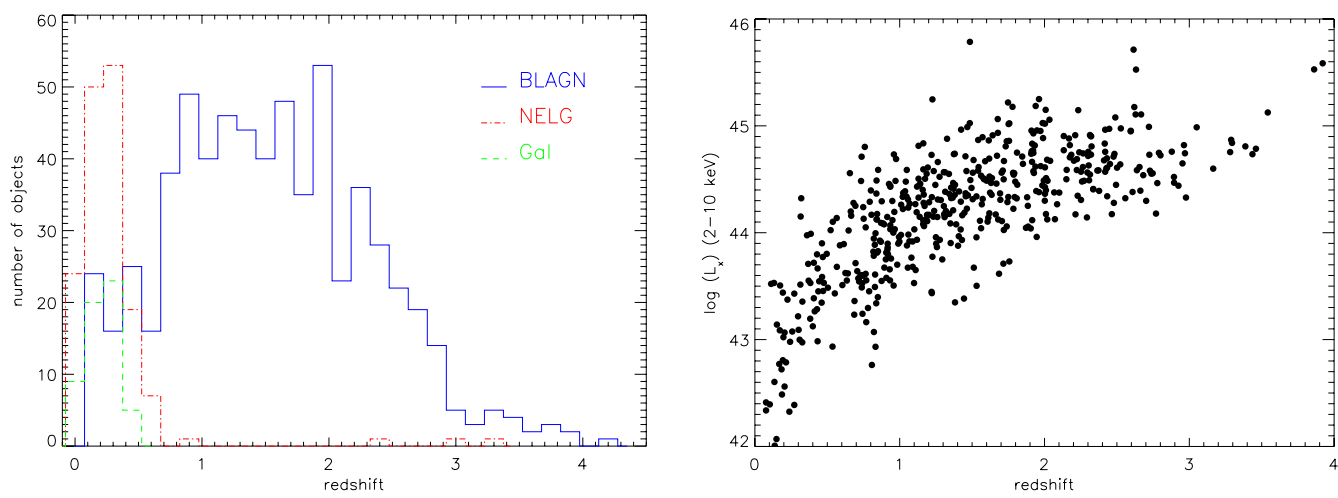

Figure 1. Left panel: redshift distributions of the different samples of extragalactic objects in the XMM-2dF Wide Angle Survey. Right panel: $2-10 \mathrm{keV}$ luminosity vs redshift for the sample of BLAGN.

\section{The XMM-2dF Wide Angle Survey}

The XMM-2dF Wide Angle Survey contains 68 XMM-Newton fields with typical exposure times of a few tens of ksec, and therefore it is a medium-depth wide angle (total sky area of $15 \mathrm{deg}^{2}$ ) survey. More than 3000 XMM-Newton serendipitously discovered objects with X-ray fluxes $>10^{-14} \mathrm{erg} \mathrm{cm}^{-2} \mathrm{~s}^{-1}$ and SUPER-COSMOS optical counterparts brighter than $\mathrm{V} \sim 21$ have been observed and reduced. At the time of this analysis 978 sources have been spectroscopically identified. Most of the objects have been identified as BLAGN, 641 (65\%) while 157 objects were found to be NELG (16\%) and 57 Galaxies $(5.8 \%)$. The sample contains also 123 Stars (12.6\%). The redshift distributions of the different types of extragalactic sources is shown in Figure 1 (left), while the $2-10 \mathrm{keV}$ luminosity versus redshift distribution of sources in shown in Figure 1 (right).

\section{Spectral analysis}

For all objects with spectroscopic identifications, X-ray spectra from each EPIC-camera and observation were extracted. The spectra were then combined to obtain a co-added MOS and pn spectrum for each source. From the 641 objects in the BLAGN sample, 496 have X-ray spectra of sufficient quality (at least 5 bins in the co-added spectra) to characterise the emission properties of the objects from an analysis of their X-ray spectra. As a starting point of our study, all spectra were fitted with a power law and an absorbed power law, both absorbed by the column density of the Galaxy.

BLAGN mean photon index The $0.2-12 \mathrm{keV}$ X-ray spectra of most BLAGN in our sample were best fitted with a simple power law. The values of the X-ray photon index were found to be near constant and consistent in most cases with the canonical value of 1.9. However, a clear dispersion on the individual values was evident as can be seen from the distribution of best fit spectral slopes in Figure 2 (left). Using a maximum likelihood analysis (see Maccacaro et al. 1988) we measured the mean continuum shape of our BLAGN to be $1.96 \pm 0.05$ with an intrinsic dispersion $\sigma=0.22 \pm 0.03$. However, for a large number of objects in our sample the best fit photon index was found to be significantly different from the mean value. We can explain this result as being due to the fact that we are not detecting all X-ray absorption present in the X-ray spectra of our sources, probably due to the low signal of some of the data. This is confirmed by the fact that the mean photon index of our objects becomes harder at the faintest soft $(0.5-2 \mathrm{keV})$ fluxes (see Figure 2), an effect that has been attributed to undetected X-ray absorption 

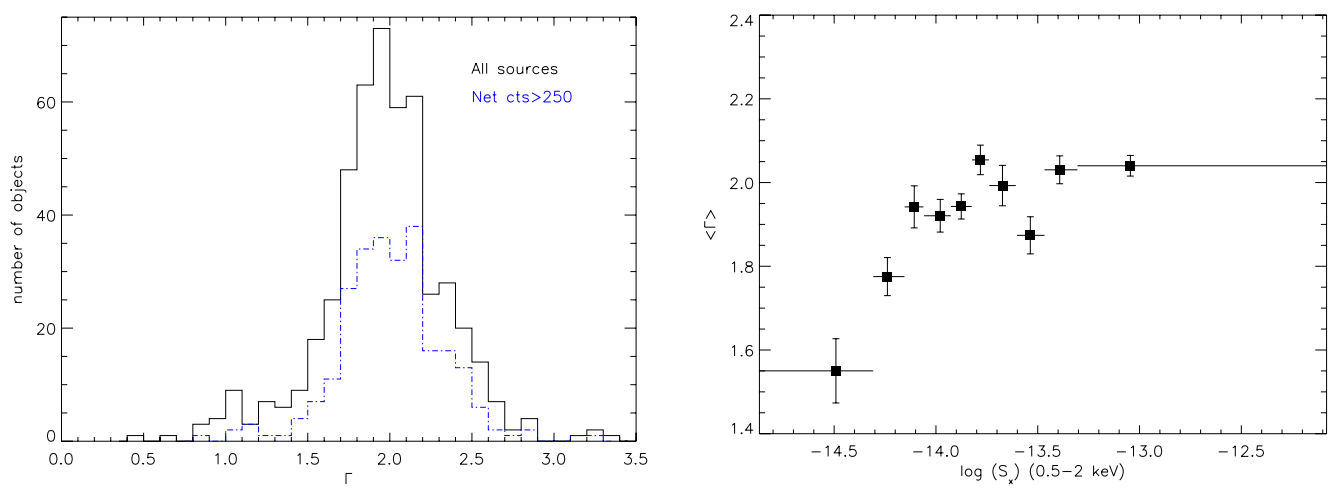

Figure 2. Left panel: distribution of best fit spectral slopes for our sample of BLAGN (solid). The corresponding distribution for the objects with best spectral quality is indicated with a dashed line. Right panel: dependence of the mean continuum shape with the soft $(0.5-2 \mathrm{keV})$ flux of the objects.
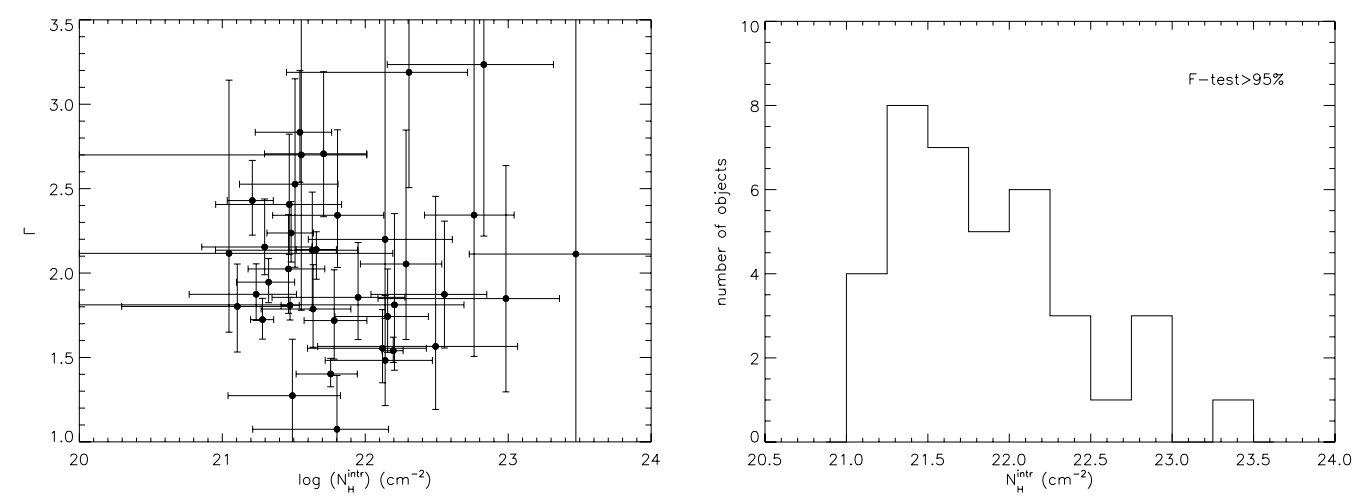

Figure 3. Left panel: correlation of the spectral photon index with X-ray absorption. Right panel: distribution of X-ray absorbing column densities (rest-frame) in our sample of BLAGN.

(Mateos et al. 2005a,b). In addition, our spectral models do not contain a soft excess component.

Intrinsic $X$-ray absorption Intrinsic X-ray absorption was detected in 38 objects $(\sim 8 \%$ of the sample) with an F-test significance $\geqslant 95 \%$. No correlation of the power law shape with the detected absorption was found as we see in Figure 3 (left). X-ray absorption in our BLAGN does not seem to occur at any particular redshift or luminosity. The distribution of absorbing column densities (rest-frame) of our sample of absorbed BLAGN is shown in Figure 3 (right). Typical absorbing column densities are $<10^{22}$ although a significant fraction of type-1 AGN have detected absorbing column densities above this value but with large uncertainties in the values. We did not find the fraction of absorbed BLAGN to depend on the X-ray luminosity of the sources or that X-ray absorption occurs at any preferred redshift.

\section{Dependence of emission properties with luminosity and redshift}

It has been previously reported that the X-ray photon index of AGN depends on the luminosity of the objects; see Dai et al. (2004). On the other hand recent spectral analyses based on sources detected with Chandra and XMM-Newton claim contradictory results on the cosmic evolution of the photon index of AGN: some suggest a hardening 

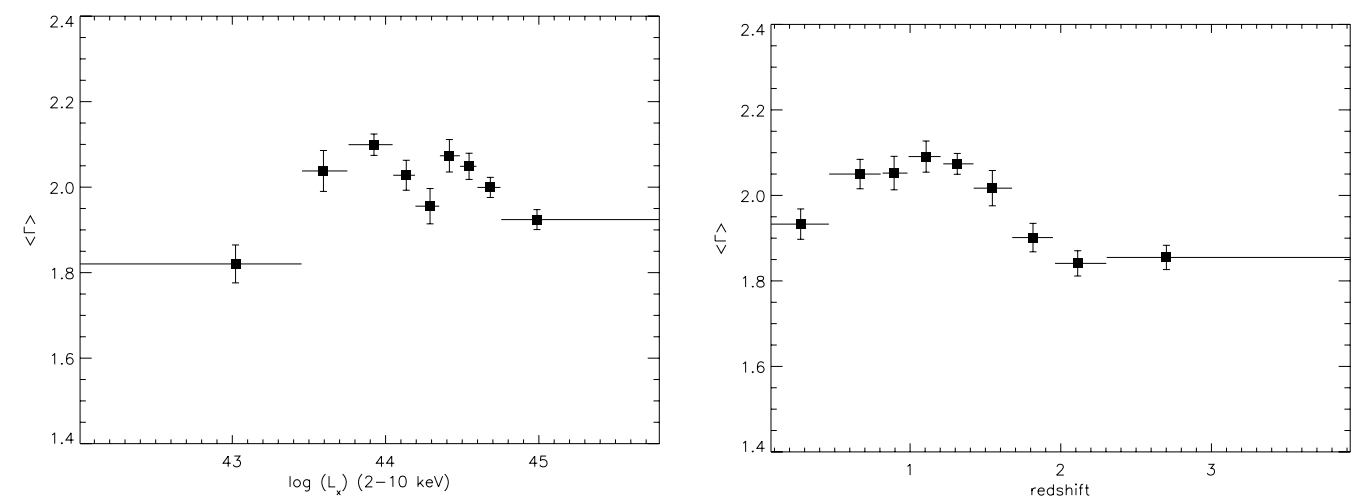

Figure 4. Left panel: dependence of the mean photon index of our BLAGN with the $2-10 \mathrm{keV}$ luminosity of the sources. Right panel: the mean photon index of our BLAGN vs redshift.
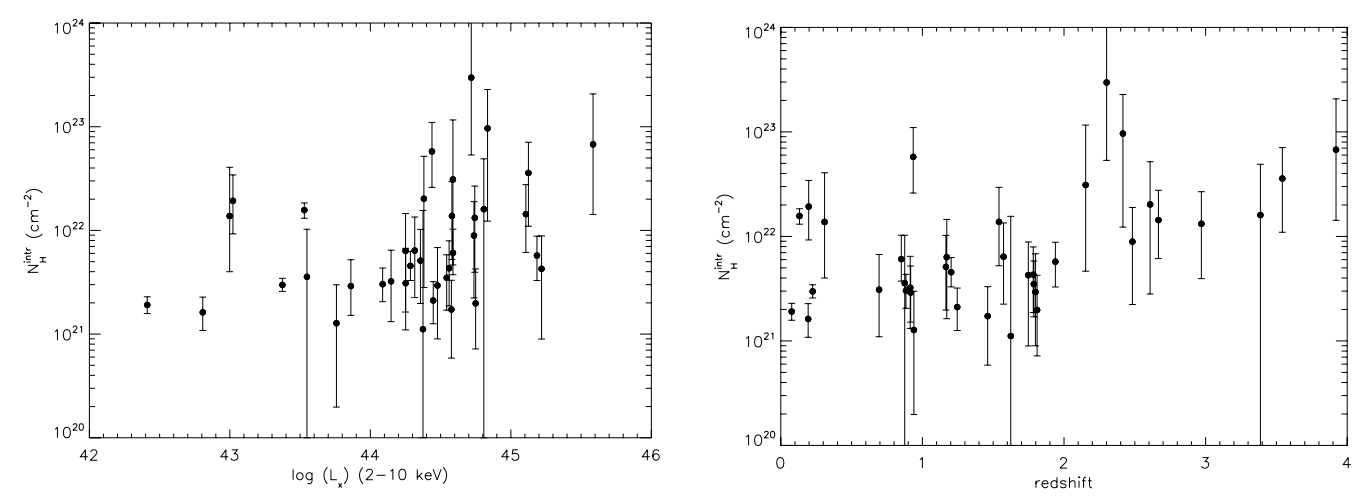

Figure 5. Left panel: dependence of X-ray absorption of our BLAGN with the $2-10 \mathrm{keV}$ luminosity of the sources. Right panel: X-ray absorption of BLAGN vs redshift.

at high redshifts (Bechtold et al. 2003) while others suggest a softening (Grupe et al. 2006) or no significant evolution (Vignali et al. 2005). We do not see any clear trend of the mean photon index of our objects with X-ray luminosity in the range of luminosities $\sim 10^{43}-10^{45} \mathrm{erg} \mathrm{s}^{-1}$ (see Figure 4). Finally we did not found evidence for a correlation of absorption and redshift/luminosity (see Figure 5) in agreement with the findings of Dwelly \& Page (2006).

\section{Acknowledgements}

This work was supported by funding from the XMM-Newton Survey Science Centre.

\section{References}

Bechtold J., Siemiginowska A., Shields J., Czerny B. et al. 2003, ApJ, 588, 119

Dai X., Chartas G., Eracleous M. \& Garmire G. P. 2004, ApJ, 605, 45

Dwelly T. \& Page M. J. 2006, MNRAS, 1122

Grupe D., Mathur S., Wilkes B. \& Osmer P. 2006, AJ, 131, 55

Maccacaro T., Gioia L. M., Wolter A. et al. 1988, ApJ, 326, 680

Mateos S., Barcons X., Carrera F. J., Ceballos M. T. et al. 2005, A\&A, 433, 855

Mateos S., Barcons X., Carrera F. J., Ceballos M. T. et al. 2005, A\&A, 444, 79

Tozzi P., Gilli R., Mainieri V., Norman C. et al. 2005, A\&A, 451, 457

Vignali C., Brandt W. N., Schneider D. P. \& Kaspi S. 2005, AJ, 129, 2519 
Richard Mushotzky: Since you show many of the optically selected broad-line objects have column densities between $10^{21}-10^{22}$, these would be normally expected highly reddened. Is this evident or absent in the optical data? That would mean a strong correction to the standard optical luminosity function data.

Silvia Mateos: We have some evidence that the absorbed objects are redder, but no firm conclusions can be made at this moment.

BriAn Boyle: Comment: Regarding the optical selection in the 2dF survey, the optical colours of low-redshift objects might have might kicked them out of the survey. This might account for the fact that you don't see an increase of absorption in the X-ray broad line AGN towards lower redshift, which seems slightly inconsistent with the picture that Günther Hasinger has proposed. 


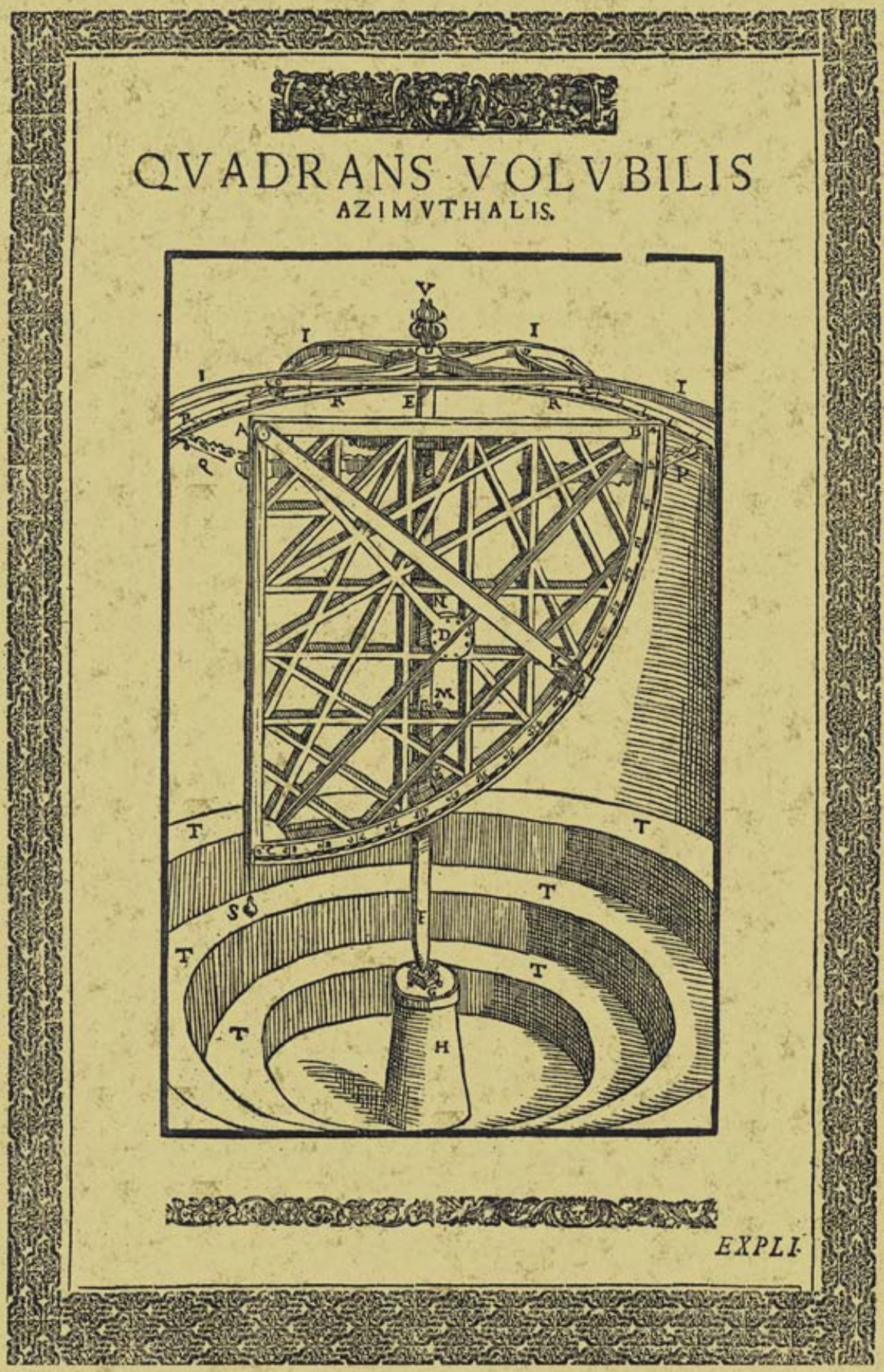

